\title{
PERIODIC SOLUTIONS OF NONLINEAR VECTOR DIFFERENCE EQUATIONS
}

\author{
M. I. GIL'
}

Received 31 January 2005; Accepted 7 September 2005

Essentially nonlinear difference equations in a Euclidean space are considered. Conditions for the existence of periodic solutions and solution estimates are derived. Our main tool is a combined usage of the recent estimates for matrix-valued functions with the method of majorants.

Copyright (c) 2006 M. I. Gil'. This is an open access article distributed under the Creative Commons Attribution License, which permits unrestricted use, distribution, and reproduction in any medium, provided the original work is properly cited.

\section{Introduction and notation}

Periodic solutions of difference equations in Euclidean and Banach spaces have been considered by many authors, see, for example, $[1-3,5-10,12]$ and the references therein. Mainly equations with separated linear parts and scalar equations were investigated. In this paper, we consider essentially nonlinear systems in a Euclidean space. We prove the existence of periodic solutions and derive the estimates for their norms.

Let $\mathbb{C}^{n}$ be the set of all complex $n$-vectors with an arbitrary norm $\|\cdot\|, I$ is the unit matrix, $R_{s}(A)$ denotes the spectral radius of a matrix $A$, and

$$
\Omega(r)=\left\{z \in \mathbb{C}^{n}:\|z\| \leq r\right\} .
$$

Consider in $\mathbb{C}^{n}$ the equation

$$
x(t+1)=B(x(t), t) x(t)+F(x(t), t) \quad(t=0,1,2, \ldots),
$$

where $F(\cdot, t)$ continuously maps $\Omega(r)$ into $\mathbb{C}^{n}$, and $B(z, t)$ are $n \times n$-matrices continuous in $z \in \Omega(r)$ and dependent on $t=0,1, \ldots$ In addition, $F(v, t)$ and $B(v, t)$ are periodic in $t$ :

$$
\begin{array}{ll}
F(z, t)=F(z, t+T) & (z \in \Omega(r) ; t=0,1, \ldots), \\
B(z, t)=B(z, t+T) & (z \in \Omega(r) ; t=0,1, \ldots)
\end{array}
$$

Hindawi Publishing Corporation Advances in Difference Equations Volume 2006, Article ID 39419, Pages 1-8 DOI 10.1155/ADE/2006/39419 
2 Periodic solutions of nonlinear vector difference equations

for some positive integer $T$. It is also assumed that there are nonnegative constants $\nu$ and $\mu$, such that

$$
\|F(z, t)\| \leq v\|z\|+\mu \quad(z \in \Omega(r), t=0,1,2, \ldots, T-1) .
$$

Denote by $\omega(r, T)$ the set of the finite sequences $h=\{v(k)\}_{k=0}^{T-1}$ whose elements $v(k)$ belong to $\Omega(r)$.

For an $h=\{v(k)\}_{k=0}^{T} \in \omega(r, T)$, put

$$
\begin{gathered}
U_{h}(t, s)=B(v(t-1), t-1) B(v(t-2), t-2) \cdots B(v(s), s), \\
U_{h}(t, t)=I \quad(0 \leq s<t \leq T)
\end{gathered}
$$

and assume that

$$
I-U_{h}(T, 0) \text { is invertible } \forall h \in \omega(r, T) .
$$

\section{Statement of the main result}

Theorem 2.1. Under conditions (1.3)-(1.6), with the notation

$$
\begin{aligned}
M(r, T):= & \sup _{h \in \omega(r, T) ; k=0, \ldots, T-1} \sum_{j=0}^{T-1}\left\|U_{h}(k, 0)\left(I-U_{h}(T, 0)\right)^{-1} U_{h}(T, j+1)\right\| \\
& +\sum_{j=0}^{k-1}\left\|U_{h}(k, j+1)\right\|
\end{aligned}
$$

suppose that

$$
M(r, T)(\nu r+\mu)<r
$$

Then system (1.2) has a T-periodic solution. Moreover, that periodic solution satisfies the estimates

$$
\max _{j=0,1, \ldots, T-1}\|x(j)\| \leq \frac{\mu M(r, T)}{1-v M(r, T)}<r .
$$

We remark that if $F(0, t) \neq 0$ for some $t$ in $\{0,1, \ldots, T-1\}$, then the solution found in the above theorem cannot be trivial.

For instance, let

$$
\|B(z, t)\| \leq q<1 \quad(z \in \Omega(r), t=0, \ldots, T-1) .
$$

Then $\left\|U_{h}(k, j)\right\| \leq q^{k-j}$ and

$$
\left\|\left(I-U_{h}(T, 0)\right)^{-1}\right\| \leq \frac{1}{1-q^{T}} .
$$


Therefore

$$
M(r, T) \leq \sum_{j=0}^{T-1} \frac{1}{1-q^{T}} q^{T-j-1}+\max _{k} \sum_{j=0}^{k-1} q^{k-j-1} \leq \sum_{j=0}^{T-1} q^{j}\left(\frac{1}{1-q^{T}}+1\right)=\frac{2-q^{T}}{1-q^{T}} \sum_{j=0}^{T-1} q^{j} .
$$

But

$$
\sum_{j=0}^{T-1} q^{j}=\frac{1-q^{T}}{1-q}
$$

Thus

$$
M(r, T) \leq \frac{2-q^{T}}{1-q} .
$$

Now Theorem 2.1 implies the following corollary.

Corollary 2.2. Under conditions (1.3)-(1.4) and (2.4), suppose that

$$
(r \nu+\mu) \frac{2-q^{T}}{1-q}<r
$$

Then system (1.2) has a T-periodic solution. Moreover that periodic solution satisfies the estimates

$$
\max _{j=0,1, \ldots, T-1}\|x(j)\| \leq \frac{\mu\left(2-q^{T}\right)}{1-q-\nu\left(2-q^{T}\right)} \leq r .
$$

\section{Proof of Theorem 2.1}

To achieve our goal, let us first consider the nonhomogeneous periodic problem

$$
\begin{gathered}
y(t+1)=B(v(t), t) y(t)+f(t), \quad t=0,1, \ldots, T-1 \\
y(0)=y(T),
\end{gathered}
$$

where $\{f(t)\}_{k=0}^{T-1}$ is a given sequence in $\mathbb{C}^{n}$ and $h=\{v(t)\} \in \omega(r, T)$. Thanks to the Variation of constants formula, solution of (3.1) is given by

$$
y(k)=U_{h}(k, 0) y(0)+\sum_{j=0}^{k-1} U_{h}(k-1, j+1) f(j), \quad k=1, \ldots, T .
$$

Thus, the periodic boundary value problem (3.1), (3.2) has a solution provided

$$
y(0)=y(T)=U_{h}(T, 0) y(0)+\sum_{j=0}^{T-1} U_{h}(T, j+1) f(j),
$$


4 Periodic solutions of nonlinear vector difference equations

or

$$
y(0)=\left(I-U_{h}(T, 0)\right)^{-1} \sum_{j=0}^{T-1} U_{h}(T, j+1) f(j),
$$

and in such a case, this solution is given by

$y(k)=U_{h}(k, 0)\left(I-U_{h}(T, 0)\right)^{-1} \sum_{j=0}^{T-1} U_{h}(T, j+1) f(j)+\sum_{j=0}^{k-1} U_{h}(k, j+1) f(j), \quad k=1, \ldots, T$,

and thus its maximum norm satisfies the inequality

$$
\max _{j=0,1, \ldots, T-1}\|y(j)\| \leq M(r, T) \max _{j=0,1, \ldots, T-1}\|f(j)\| .
$$

Let us consider the nonlinear periodic problem (1.2), (3.2).

Lemma 3.1. Under conditions (1.4), (1.6), and (2.2), the periodic problem (1.2), (3.2) has at least one solution $\{x(t)\}_{t=0}^{T} \in \omega(r, T)$. Moreover, that solution satisfies estimates (2.3).

Proof. For an arbitrary $h=\{v(t)\} \in \omega(r, T)$, define a mapping $Z$ by

$$
\begin{aligned}
(Z h)(k)= & U_{h}(k, 0)\left(I-U_{h}(T, 0)\right)^{-1} \sum_{j=0}^{T-1} U_{h}(T, j+1) F(v(j), j) \\
& +\sum_{j=0}^{k-1} U_{h}(k, j+1) F(v(j), j), \quad k=0, \ldots, T-1 .
\end{aligned}
$$

Due to (2.2),

$$
\begin{aligned}
\max _{j=0,1, \ldots, T-1}\|(Z h)(j)\| & \leq \max _{t=0, \ldots, T-1}\|F(v(t), t)\| M(r, T) \\
& \leq\left(\nu \max _{j=0, \ldots, T-1}\|v(j)\|+\mu\right) M(r, T) \leq \nu r+\mu .
\end{aligned}
$$

So $Z$ continuously maps $\omega(r, T)$ into itself. By Browder's fixed point theorem, $Z$ has a fixed point $x \in \omega(r, T)$, cf. [11]. It is easily checked that the point is the desired solution of problem (1.2), (3.2).

Furthermore, if $\{x(t)\}_{t=0}^{T} \in \omega(r, T)$ is a solution of (1.2), (3.2), then in view of (3.7) and (1.4), we will have the relations

$$
\max _{j=0,1, \ldots, T-1}\|x(j)\| \leq \max _{t=0,1, \ldots, T-1}\|F(x(t), t)\| M(r, T) \leq\left(\nu \max _{j=0, \ldots, T}\|x(j)\|+\mu\right) M(r, T),
$$

which implies (2.3), since under $(2.2) \nu M(r, T)<1$. The proof is complete.

Assertion of Theorem 2.1 follows from the previous lemma and the periodicity of $F(\cdot, t)$ and $B(\cdot, t)$ in $t$. 


\section{Systems with linear majorants}

In this section and the next one it is assumed that the norm is ideal. That is the vectors $z=\left(z_{k}\right)_{k=1}^{n}$ and $|z|=\left(\left|z_{k}\right|\right)_{k=1}^{n}$ have the same norm. For example,

$$
\|z\|=\|z\|_{p}=\left[\sum_{k=1}^{n}\left|z_{k}\right|^{p}\right]^{1 / p} \quad(1 \leq p<\infty) .
$$

Let there be a variable matrix $W(t)=\left(w_{j k}(t)\right)_{j, k=1}^{n} t=0, \ldots, T$ independent of $z$ with nonnegative entries, such that the relation

$$
|B(z, t)| \leq W(t) \quad(z \in \Omega(r), t=0, \ldots, T-1)
$$

is valid with a positive $r<\infty$. Then we will say that $B(\cdot, t)=\left(b_{\{j k\}}(\cdot, t)\right)_{j, k=1}^{n}$ has in $\Omega(r)$ the linear majorant $W(t)$.

Inequality (4.2) means that

$$
\left|b_{j k}(z, t)\right| \leq w_{j k}(t) \quad(j, k=1, \ldots, n ; z \in \Omega(r), t=1,2, \ldots, T) .
$$

Let us introduce the equation

$$
y(t+1)=W(t) y(t) \quad(t=1,2, \ldots) .
$$

Lemma 4.1. Let $B(\cdot, t)$ have a linear majorant $W(t)$ in the ball $\Omega(r)$. Then

$$
\left\|U_{h}(t, s)\right\| \leq\|V(t, s)\| \quad(h \in \omega(r, T), 0 \leq s<t \leq T-1)
$$

where $V(t, s)=W(t-1) W(t-2) \cdots W(s)$.

Proof. Clearly,

$$
\left\|U_{h}(t, s)\right\|=\|B(v(t-1), t-1) \cdots B(v(s), s)\| \leq\|W(t-1) \cdots W(s)\| .
$$

This proves the result.

Furthermore, assume that the spectral radius of $V(T, 0)$ is less than one. Then the matrix $I-V(T, 0)$ is positively invertible. Put

$$
m(W, T):=\sup _{k=0, \ldots, T-1} \sum_{j=0}^{T-1}\left\|V(k, 0)(I-V(T, 0))^{-1} V(T, j+1)\right\|+\sum_{j=0}^{k-1}\|V(k, j+1)\| .
$$

Now Theorem 2.1 implies the following theorem.

THEOREM 4.2. Under conditions (1.3)-(1.4) and (4.2) assume that the evolution operator of (4.4) satisfy the inequality $R_{s}(V(T, 0))<1$. In addition, suppose that

$$
(r \nu+\mu) m(W, T)<r .
$$


6 Periodic solutions of nonlinear vector difference equations

Then system (1.2) has a T-periodic solution. Moreover, that periodic solution satisfies the estimates

$$
\max _{j=0,1, \ldots, T-1}\|x(j)\| \leq \frac{\mu m(W, T)}{1-\nu m(W, T)} \leq r .
$$

\section{Systems with constant majorants}

Assume that in (4.2) $W(t) \equiv W_{0}$ is a constant matrix. Then we will say that $B(h, t)$ has in set $\Omega(r)$ the constant majorant $W(t)$. In this case $V(t, s)=W_{0}^{t-s}$. Set

$$
m\left(W_{0}, T\right)=\max _{k=0, \ldots, T-1}\left\{\left\|W_{0}^{k}\left(I-W_{0}^{T}\right)^{-1}\right\|+1\right\} \sum_{j=0}^{T-1}\left\|W_{0}^{j}\right\| .
$$

Now Theorem 4.2 yields the following theorem.

Theorem 5.1. Under conditions (1.3)-(1.4) assume that $B(\cdot, s)$ has in $\Omega(r)$ a constant majorant $W_{0}$, and $R_{s}\left(W_{0}\right)<1$. In addition, suppose that

$$
(\mu+r \nu) m\left(W_{0}, T\right)<r .
$$

Then system (1.2) has a T-periodic solution. Moreover, that periodic solution satisfies the estimates

$$
\max _{j=0,1, \ldots, T-1}\|x(j)\| \leq \frac{\mu m\left(W_{0}, T\right)}{1-\nu m\left(W_{0}, T\right)}<r .
$$

Let us derive an estimate for $m\left(W_{0} ; T\right)$ in terms of the eigenvalues and the Frobenius norm of $W_{0}$ as follows. Let $\|\cdot\|_{2}$ be the Euclidean norm in $\mathbb{C}^{n}$, and $A$ be an $n \times n$-matrix. Let $\lambda_{1}(A), \ldots, \lambda_{n}(A)$ be the eigenvalues of $A$ including their multiplicities. We will make use of the following quantity

$$
g(A)=\left\{N^{2}(A)-\sum_{i=1}^{n}\left|\lambda_{i}(A)\right|^{2}\right\}^{1 / 2},
$$

where $N(A)$ is the Frobenius (Hilbert-Schmidt) norm of $A$, that is, $N^{2}(A)=\operatorname{Trace}\left(A A^{*}\right)$. Below we give simple estimates for $g(A)$.

Next, we recall that the following estimates are valid:

$$
\begin{gathered}
\left\|A^{m}\right\|_{2} \leq \sum_{k=0}^{n-1} R_{s}^{m-k}(A) g^{k}(A) \frac{C_{m}^{k}}{\sqrt{k !}} \quad(m=0,1, \ldots), \\
\left\|(A-\lambda I)^{-1}\right\|_{2} \leq \sum_{k=0}^{n-1} \frac{g^{k}(A)}{\sqrt{k !} \rho^{k+1}(A, \lambda)},
\end{gathered}
$$

where

$$
C_{m}^{k}=\frac{m !}{(m-k) ! k !}
$$


and $\rho(A, \lambda)$ is the distance between $\lambda \in \mathbb{C}$ and the spectrum of $A$. Estimates (5.5) and (5.6) are proved in [4, pages 12 and 21]. Thus,

$$
\left\|W_{0}^{m}\right\|_{2} \leq \theta_{m}\left(W_{0}\right), \quad m=0,1,2, \ldots,
$$

where

$$
\theta_{m}\left(W_{0}\right)=\sum_{k=0}^{n-1} R_{s}^{m-k}\left(W_{0}\right) g^{k}\left(W_{0}\right) \frac{C_{m}^{k}}{\sqrt{k !}} .
$$

Furthermore, due to (5.6)

$$
\left\|\left(W_{0}^{T}-I\right)^{-1}\right\|_{2} \leq v\left(T, W_{0}\right),
$$

where

$$
v\left(T, W_{0}\right)=\sum_{k=0}^{n-1} \frac{g^{k}\left(W_{0}^{T}\right)}{\sqrt{k !}\left(1-R_{s}^{T}\left(W_{0}\right)\right)^{k+1}} .
$$

Then

$$
m\left(W_{0} ; T\right) \leq \widetilde{M}\left(W_{0} ; T\right)
$$

where

$$
\widetilde{M}\left(W_{0} ; T\right):=\left\{v\left(T, W_{0}\right) \max _{k=0, \ldots, T-1} \theta_{k}\left(W_{0}\right)+1\right\} \sum_{j=0}^{T-1} \theta_{j}\left(W_{0}\right) .
$$

Under the condition, $R_{s}\left(W_{0}\right)<1$ we have

$$
\max _{k=0, \ldots, T-1} \theta_{k}\left(W_{0}\right) \leq 2^{T-1} \sum_{k=0}^{n-1} \frac{g^{k}\left(W_{0}\right)}{\sqrt{k !}} .
$$

Note also that $g\left(W_{0}^{T}\right) \leq N^{T}\left(W_{0}\right)$. Moreover, if $A$ is a normal matrix: $A A^{*}=A^{*} A$, then $g(A)=0$. The following inequalities are also true

$$
\begin{gathered}
g^{2}(A) \leq N^{2}(A)-\left|\operatorname{Trace} A^{2}\right|, \\
g^{2}(A) \leq \frac{1}{2} N^{2}\left(A^{*}-A\right),
\end{gathered}
$$

\section{cf. [4, Section 2.1].}

Now Theorem 5.1 implies the following theorem.

Theorem 5.2. Under conditions (1.3)-(1.4), assume that $B(\cdot, t)$ has in $\Omega(r)$ a constant majorant $W_{0}$ and $R_{s}\left(W_{0}\right)<1$. In addition, let

$$
(\mu+r \nu) \widetilde{M}\left(W_{0} ; T\right)<r
$$


Then system (1.2) has a T-periodic solution. Moreover, that periodic solution satisfies the estimates

$$
\max _{j=0,1, \ldots, T-1}\|x(j)\| \leq \frac{\mu \widetilde{M}\left(W_{0}, T\right)}{1-v \widetilde{M}\left(W_{0}, T\right)} \leq r .
$$

As an example, let $W_{0}$ be a normal matrix, then $g\left(W_{0}\right)=0, \theta_{m}\left(W_{0}\right)=R_{s}^{m}\left(W_{0}\right) \leq 1$ and

$$
\widetilde{M}\left(W_{0}, T\right)=\frac{1}{1-R_{s}^{T}\left(W_{0}\right)}
$$

Now we can directly apply the previous theorem.

\section{Acknowledgment}

This research was supported by the Kamea Fund of the Israel Ministry of Science and Technology.

\section{References}

[1] S. S. Cheng and G. Zhang, Positive periodic solutions of a discrete population model, Functional Differential Equations 7 (2000), no. 3-4, 223-230.

[2] S. Elaydi and S. Zhang, Stability and periodicity of difference equations with finite delay, Funkcialaj Ekvacioj. Serio Internacia 37 (1994), no. 3, 401-413.

[3] M. I. Gil', Periodic solutions of abstract difference equations, Applied Mathematics E-Notes 1 (2001), 18-23.

[4] _ Operator Functions and Localization of Spectra, Lecture Notes in Mathematics, vol. 1830, Springer, Berlin, 2003.

[5] M. I. Gil' and S. S. Cheng, Periodic solutions of a perturbed difference equation, Applicable Analysis 76 (2000), no. 3-4, 241-248.

[6] M. I. Gil', S. Kang, and G. Zhang, Positive periodic solutions of abstract difference equations, Applied Mathematics E-Notes 4 (2004), 54-58.

[7] A. Halanay, Solutions périodiques et presque-périodiques des systèmes d'équations aux différences finies, Archive for Rational Mechanics and Analysis 12 (1963), 134-149.

[8] A. Halanay and V. Rǎsvan, Stability and Stable Oscillations in Discrete Time Systems, Advances in Discrete Mathematics and Applications, vol. 2, Gordon and Breach Science, Amsterdam, 2000.

[9] G. P. Pelyukh, On the existence of periodic solutions of discrete difference equations, Uzbekskiı̌ Matematicheskiĭ Zhurnal (1995), no. 3, 88-90 (Russian).

[10] Kh. Turaev, On the existence and uniqueness of periodic solutions of a class of nonlinear difference equations, Uzbekskiŭ Matematicheskiǐ Zhurnal (1994), no. 2, 52-54 (Russian).

[11] E. Zeidler, Nonlinear Functional Analysis and Its Applications. I. Fixed-point Theorems, Springer, New York, 1986.

[12] R. Y. Zhang, Z. C. Wang, Y. Chen, and J. Wu, Periodic solutions of a single species discrete population model with periodic harvest/stock, Computers \& Mathematics with Applications 39 (2000), no. 1-2, 77-90.

M. I. Gil': Department of Mathematics, Ben Gurion University of the Negev, P.O. Box 653,

Beer-Sheva 84105, Israel

E-mail address: gilmi@cs.bgu.ac.il 